\title{
Possible Mechanisms of Action of SGLT2 Inhibitors in Heart Failure
}

\author{
Camila Nicolela Geraldo Martins, ${ }^{1}$ Adriana Aparecida Bau, ${ }^{1}$ Luis Miguel da Silva, ${ }^{1}$ Otavio Rizzi Coelho-Filho ${ }^{1}$ [D \\ Faculdade de Ciências Médicas - Universidade Estadual de Campinas, 1 Campinas, SP - Brazil
}

\begin{abstract}
In mid-2013, sodium-glucose co-transporter 2 (SGLT2) inhibitors such as canagliflozin, dapagliflozin, and empagliflozin were introduced as possible treatments for type 2 diabetes mellitus (DM2). Cardiovascular safety studies demonstrated that not only were SGLT2 inhibitors safe, but they were also associated with a significant reduction in cardiovascular mortality and heart failure (HF) outcomes including hospitalizations. These findings encouraged the development of clinical trials aimed specifically at investigating the effects of this new drug class on HF with reduced ejection fraction (EF), including the DAPA-HF and EMPEROR-reduced studies. The demonstration of beneficial effects of SGLT2 inhibitors on the reduction of cardiovascular events in treated patients with symptomatic HF and reduced left ventricular EF (LVEF $<40 \%$ ) resulted in the extension of SGLT2 inhibitor indication to patients with symptomatic HF with reduced EF. The mechanisms behind these beneficial effects of SGLT2 inhibitors are not entirely known. In this review, we analyze several current hypotheses for the cardioprotective effects of SGLT2 inhibitors, including reduced blood pressure, increased natriuresis, improved energy metabolism, prevention of inflammation, weight loss, improved glycemic control, inhibition of the sympathetic nervous system, prevention of myocardial remodeling, prevention of ischemia/ reperfusion injury, inhibition of $\mathrm{Na}+/ \mathrm{H}+$ channels, increased autophagy and lysosomal degradation, SGLT1 inhibition, reduced hyperuricemia, reduced epicardial fat, increased erythropoietin levels, increased progenitor/precursor cells, reduced oxidative stress, and improved vascular function.
\end{abstract}

\section{Introduction}

As the global number of patients with type 2 diabetes mellitus (DM2) approaches 500 million, that of patients with heart failure (HF) reaches 64 million. ${ }^{1-3} \mathrm{HF}$ and DM2 often co-occur, resulting in a worse prognosis for both conditions. Records suggest that DM2 can be present in up to $40 \%$ of patients with HF and reduced ejection fraction (EF) and up to $45 \%$ of those with preserved EF. Additionally, patients

\section{Keywords}

Heart Failure; Diabetes Mellitus; Sodium-Glucose Transporter 2 Inhibitors.

\section{Otávio Rizzi Coelho-Filho •}

Disciplina de Cardiologia, Departamento de Medicina Interna,Hospital das Clínicas da Universidade Estadual de Campinas, UNICAMP - Rua Vital Brasil, 251. Postal Code 13083-888, Cidade Universitária "Zeferino Vaz", Campinas, SP - Brazil Email: orcfilho@unicamp.br

Manuscript received April 28, 2021, revised manuscript May 26, 2021, accepted June 08, 2021 with DM2 are 2.5 to 5 times more likely to develop HF and $30 \%$ more likely to require hospitalization for $\mathrm{HF}^{4}$ The mechanisms involved in the association of DM2 and HF are multifactorial and include diastolic left ventricular (LV) dysfunction, metabolic alterations such as lipotoxicity, renin-angiotensin-aldosterone system activation, oxidative stress, microcirculatory dysfunction, endothelial dysfunction, dysautonomia, and altered myocardial calcium cycling. ${ }^{4,5}$ Most treatments for DM2 involve increasing the activity of beta cells, insulin reposition, and the restoration of insulin sensitivity; pharmacological treatments for HF with reduced $\mathrm{EF}$, on the other hand, involve the use of beta-blockers, reninangiotensin-aldosterone system inhibitors, neprilysin inhibitors, and mineralocorticoid receptor antagonists. ${ }^{2-4,6}$ In mid-2013, sodium-glucose co-transporter 2 (SGLT2) inhibitors such as canagliflozin, dapagliflozin, and empagliflozin were introduced as possible treatments for DM2. Surprisingly, the cardiovascular safety studies originally proposed by North-American and European regulatory agencies to ensure the safety of new antidiabetic drugs found that not only were SGLT2 inhibitors safe, but they were also associated with a significant reduction in cardiovascular mortality, and especially with HF outcomes. ${ }^{7-9}$ The cardiovascular safety studies of SGLT2 inhibitors and their findings on the reduction of HF outcomes, specifically hospitalization for HF, encouraged the initiation of clinical trials dedicated to the effects of this new drug class on $\mathrm{HF}$ with reduced EF. ${ }^{10,11}$ More recently, the beneficial effects of SGLT2 inhibitors on the reduction of cardiovascular events in adequately treated patients with symptomatic HF (New York Heart Association [NYHA] functional class $>/=$ II) with reduced EF ( $<40 \%)$ led the United States Food and Drug Administration (FDA) and the Brazilian Health Regulatory Agency (Agência Nacional de Vigilância Sanitária; Anvisa) to extend the indication of SGLT2 inhibitors to the treatment of symptomatic HF with reduced EF. ${ }^{1,4,6}$ SGLT2 receptors are present in the proximal convoluted tubules of the kidney and mediate glucose uptake, accounting for $90 \%$ of renal glucose reabsorption. The inhibition of SGLT2 receptors leads to a reduction in their ability to reabsorb glucose, promoting its excretion in urine and leading to a reduction in plasma glucose levels. This effect is independent of insulin secretion so that this drug class is unlikely to cause hypoglycemia. ${ }^{12}$ Although the reduction in cardiovascular events in patients with symptomatic HF and reduced EF after adjunctive treatment with dapaglifozin ${ }^{10}$ and empaglifozin ${ }^{11}$ has been demonstrated in rigorous clinical trials, the mechanism of action of this drug class in the treatment of HF has not been fully elucidated. This review aims to discuss the main possible mechanisms of action of SGLT2 inhibitors in HF.

\section{Historical background}

The need for cardiovascular safety testing of the three main SGLT2 inhibitors was especially emphasized by the

DOI: https://doi.org/10.36660/abchf.20210007 
FDA after studies showed that thiazolidinediones ${ }^{13-15}$ and saxagliptin ${ }^{16,17}$ could increase the risk of HF outcomes and especially hospitalization. The first clinical trial to establish the cardiovascular safety of SGLT2 inhibitors was the EMPA-REG OUTCOME study, published in 2015. ${ }^{9}$ This was a randomized, double-blind, placebo-controlled clinical trial of patients with established cardiovascular disease and DM2, who were randomly allocated to empagliflozin or placebo. The study involved 7020 patients, 4687 in the treatment group and 2333 in the placebo group, followed for a mean period of 3.1 years. The primary outcomes were cardiovascular death, nonfatal myocardial infarction (MI), and nonfatal stroke. The study yielded statistically significant findings on the safety and superiority of empagliflozin, with a $32 \%$ relative risk reduction for all-cause mortality and a 35\% relative risk reduction in hospitalization for $\mathrm{HF}$, although the rates of atherothrombotic events such as $\mathrm{MI}$ and stroke did not differ between groups. ${ }^{9}$ The CANVAS ${ }^{8}$ study published in 2017 had the same objective, and included 10142 patients with DM2 and high cardiovascular risk randomly allocated to canagliflozin or placebo and followed for a mean of 3.9 years. Primary outcome rates (all-cause cardiovascular mortality, nonfatal MI, or stroke) were significantly lower in the canagliflozin group, occurring in 26.9 participants per 1000 patient-years as compared to 31.5 per 1000 patient-years in the placebo group. The study also identified possible renal benefits such as reduced albuminuria progression, improved glomerular filtration rates, and reduced renal replacement therapy and renal mortality. ${ }^{8}$

The year 2019 saw the publication of the largest and longest follow-up study of cardiovascular outcomes in this drug class: the DECLARE-TIMI 58, ${ }^{7}$ which investigated the effects of dapagliflozin on cardiovascular events. This was also a randomized clinical trial involving 17160 patients with DM2 and established atherosclerotic disease or multiple risk factors for its development. Patients were randomly allocated to $10 \mathrm{mg} /$ day dapagliflozin or placebo and followed for a mean period of 4.2 years. The two primary effectiveness outcomes were MACE (a composite of cardiovascular death, MI, or ischemic stroke) and a composite of cardiovascular death and hospitalization for HF. The use of dapagliflozin resulted

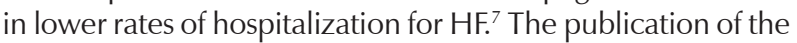
three aforementioned studies clarified the benefits of SGLT2 inhibitors on cardiovascular outcomes in patients with DM2. However, since few patients in the EMPA-REG OUTCOME, CANVAS, and DECLARE-TIMI 58 studies had a diagnosis of $\mathrm{HF}$ at the start of these investigations, dedicated studies were developed to evaluate the possible benefits of these drugs in patients with established HF. A randomized, double-blind clinical trial on patients with chronic HF with reduced EF with or without DM2 was developed to evaluate the effects of 10mg dapagliflozin once daily relative to placebo addition to standard treatment for HF. The primary outcome was a composite of cardiovascular death, hospitalization for HF, or urgent-care visits for HF. The study revealed a significant reduction in the occurrence of the primary outcome, which was reported in $16.2 \%$ of the dapagliflozin group and $21.2 \%$ of the placebo group. ${ }^{7}$ Similar findings were obtained with empagliflozin, ${ }^{11}$ which also led to a significant reduction in the occurrence of major cardiovascular events in a population of patients with symptomatic HF and reduced EF, corroborating the benefits of this class of medications in the treatment of symptomatic HF with reduced EF.

\section{Possible mechanisms of action of SGLT2 inhibitors in heart failure}

As previously mentioned, SGLT2 inhibitors, though initially developed for the treatment of DM2, have demonstrated clear benefits on cardiovascular outcomes, especially those related to $\mathrm{HF}$ with reduced $\mathrm{EF}$, in addition to kidney outcomes in patients with or without diabetes. ${ }^{18}$ Though the mechanisms through which SGLT2 inhibitors affect HF with reduced EF have not been completely elucidated, experimental studies have suggested several possible explanations for this association (Figure 1). These include the following: 1) reduced blood pressure; 2) increased natriuresis; 3 ) improved energy metabolism; 4) prevention of inflammation; 5) weight loss; 6) improved glycemic control; 7) inhibition of the sympathetic nervous system; 8) prevention of myocardial remodeling; 9) prevention of ischemia/reperfusion injury; 10) inhibition of $\mathrm{Na}+/ \mathrm{H}+$ channels; 11$)$ increased autophagy and lysosomal degradation, SGLT1 inhibition; 12) reduced hyperuricemia; 13) reduced epicardial fat; 14) increased erythropoietin (EPO) levels; 15) increased progenitor/precursor cells; 16) reduced oxidative stress; and 17) improved vascular function. Therefore, several hypotheses have been put forward to explain the effects of these drugs. The main ones will be discussed in the following sections.

\section{Weight loss}

Renal glucose excretion during treatment with SGLT2 inhibitors results in a substantial caloric loss. This results in a significant loss of body weight as fatty acids are mobilized from adipose tissue. ${ }^{18-20}$ Clinical trials have demonstrated a significant reduction in body weight in patients treated with SGLT2 inhibitors. ${ }^{21}$ While this weight loss may contribute to the beneficial effects of SGLT2 inhibitors, other mechanisms must also be involved since the effects of weight loss measures on HF severity have been far smaller than those observed with SGLT2 inhibitors. Therefore, weight loss is unlikely to be the main mechanism through which SGLT2 inhibitors affect HF outcomes. Furthermore, the effects of SGLT2 inhibitors on weight loss are moderate and tend to decrease over time due to counterregulatory mechanisms such as increased energy intake triggered to maintain body weight. ${ }^{22}$

\section{Improved glucose control}

Although SGLT2 inhibitors are effective at reducing glucose levels, this is unlikely to be directly associated with the benefits of this medication class on HF. Hyperglycemia alone is a weak risk factor for cardiovascular illness. ${ }^{23}$ Additionally, the rapid efficacy of these investigations (observed days after treatment initiation) speaks against an association between glucose reduction and improved cardiovascular outcomes. The differences in glycemic control and cardiovascular outcomes reported in previous clinical 


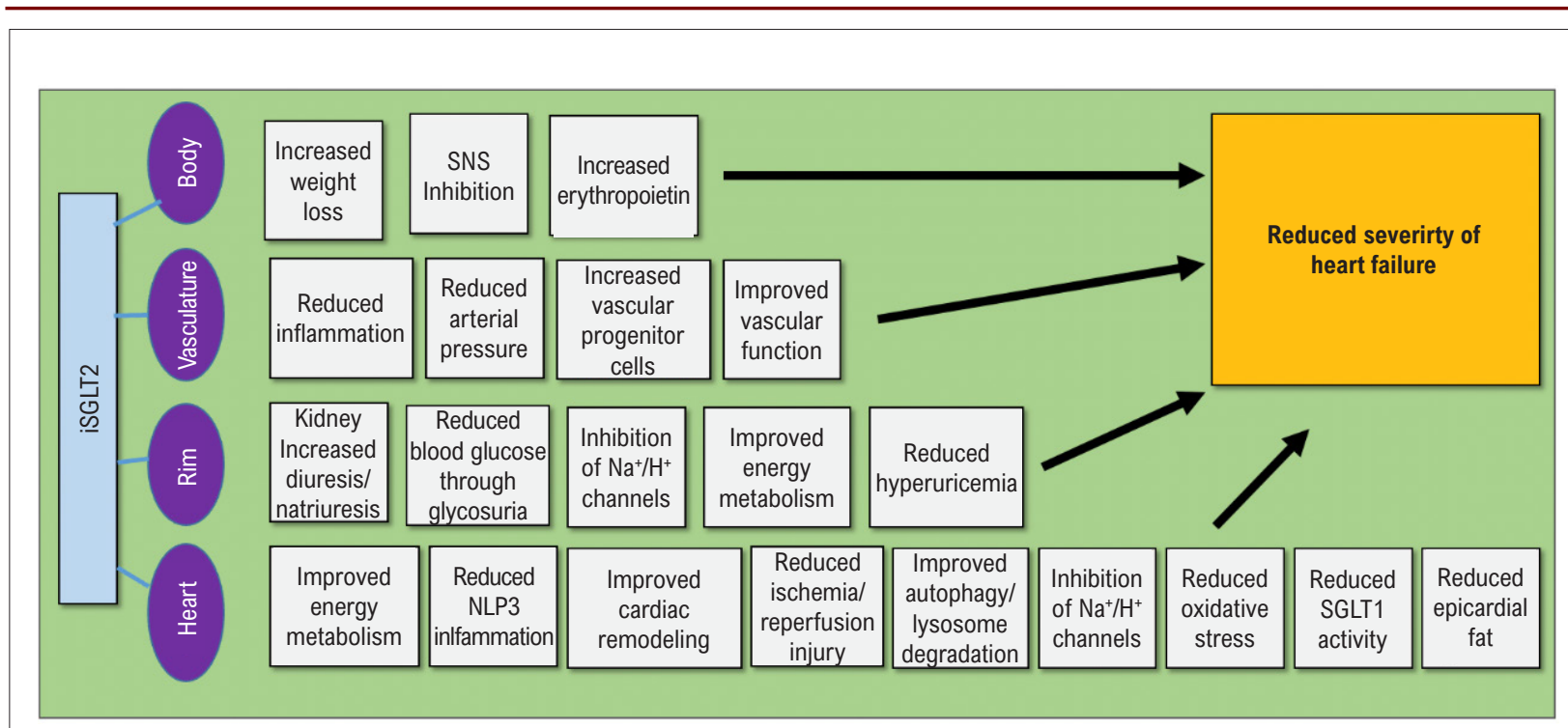

Figure 1 - Possible mechanisms through which SGLT2 inhibitors improve heart failure outcomes. iSGLT2: sodium-glucose co-transporter 2 inhibitors; SNS: sympathetic nervous system; NLRP3: nucleotide-binding oligomerization domain, leucine-rich repeat, and pyrin domain-containing; SGLT1: sodium-glucose co-transporter 1.

trials have been small, and post hoc analyses of these studies suggest that baseline glycated hemoglobin and changes in $\mathrm{HbA1c}$ are not necessarily associated with the effects of treatment with SGLT2 inhibitors. ${ }^{23}$ This was confirmed in the DAPA-HF study, which found that dapagliflozin was similarly effective in individuals with and without diabetes. ${ }^{10}$ In patients without diabetes, its effectiveness was similar to that observed in individuals with prediabetes or reduced glucose tolerance relative to normoglycemic participants. Similar findings were obtained in a study of empagliflozin, another SGLT2 inhibitor, which proved effective in the treatment of $\mathrm{HF}$ with reduced $\mathrm{EF}$ regardless of the presence of DM2. ${ }^{11}$ Polynomial analyses in the DAPA-HF study demonstrated that baseline glycated hemoglobin was unrelated to the positive effects of dapagliflozin on HF and mortality. ${ }^{10}$ Additionally, in experimental models of HF, benefits of SGLT2 inhibition have been observed regardless of whether diabetes or hyperglycemia is present. ${ }^{24,25}$

\section{Blood pressure reduction}

Hypertension is an important modifiable risk factor for HF. As such, it has been suggested that some of the beneficial effects of SGLT2 inhibitors on HF may be explained by their effects on blood pressure since SGLT2 inhibitors are known to reduce these values. ${ }^{26}$ Although the mechanisms behind the antihypertensive activity of SGLT2 inhibition are not entirely clear, these effects are likely mediated by the osmotic and diuretic effects of SGLT2 inhibitors caused by the inhibition of sodium reabsorption in the proximal tubules of the kidney. SGLT2 inhibition may increase urinary sodium excretion by 30 to $60 \%{ }^{26,27}$ The anti-hypertensive effects of SGLT2 inhibitors are larger than those of thiazide diuretics when used in combination with beta-blockers or calcium antagonists. ${ }^{28,29}$ By decreasing blood pressure, SGLT2 inhibitors may decrease the cardiac post-load, reducing cardiac overload and improving efficiency. This could be the mechanism through which SGLT2 inhibitors affect HF outcomes. However, the effects of SGLT2 inhibition on blood pressure reduction are modest and unlikely to entirely explain the beneficial effects of this drug on cardiovascular and kidney function. Additionally, reduced blood pressure is expected to have a greater effect on stroke rates relative to other cardiovascular outcomes, which was not observed in the EMPA-REG OUTCOME study. ${ }^{9}$ Lastly, in the DAPA-HF trial, blood pressure reductions were modest and unlikely to be related to a significant reduction in cardiovascular events. ${ }^{10}$

\section{Increased natriuresis}

SGLT2 inhibitors promote natriuresis and glycosuria, and it has been suggested that osmotic diuresis may improve HF outcomes. Analyses from the EMPA-REG OUTCOME study suggested that hemoconcentration (presumably secondary to volume contraction) was responsible for nearly $50 \%$ of the cardiovascular benefits observed in this investigation. ${ }^{9}$ Yet the benefits of SGLT2 inhibitors are unlikely to be explained by diuresis alone since other diuretic measures have not been associated with a reduction of cardiovascular events in HF studies. It has been suggested that SGLT2 inhibitors may differ from classical diuretics given their peculiar mechanism of action and, especially, their effects on the reduction of interstitial fluid. In a study comparing dapagliflozin and hydrochlorothiazide, for instance, reduced plasma volume and increased erythrocyte mass were observed with the former but not the latter. ${ }^{30}$ When compared to a loop diuretic (bumetanide), dapagliflozin was associated with a greater 
reduction in interstitial fluid rather than blood volume. ${ }^{31}$ It has therefore been suggested that SGLT2 inhibition may have differential effects on interstitial fluid (vs. intravascular volume) which can limit the neurohumoral stimulation that occurs in response to the contraction of intravascular volume caused by traditional diuretics.

\section{Reduced sympathetic stimulus}

SGLT2 acts by stimulating the sympathetic nervous system. The effects of hyperstimulation of this system can include arterial stiffening and, consequently, chronic elevation of blood pressure, ultimately leading to vascular and kidney damage. Both effects are directly related to poorer HF outcomes. ${ }^{32}$ SGLT2 inhibitors are associated with a reduced sympathetic tone, and the ability to induce vasodilation by activating voltage-gated potassium channels and protein kinase $G$, which are crucial for the regulation of vascular tone. Additionally, they improve endothelial function and arterial stiffness. ${ }^{12}$ The sympathetic nervous system, ${ }^{33,34}$ in turn, plays a crucial role in blood pressure control and the physiopathology of hypertension, and its activation may be related to the development of HF. ${ }^{35,36}$ Several experimental ${ }^{37}$ and clinical $^{38}$ studies have suggested that SGLT2 inhibitors reduce the activation of the sympathetic nervous system. Though the exact mechanisms behind HF risk reduction and the beneficial effects of SGLT2 inhibitors are not fully known, some hypotheses are supported by stronger evidence, such as the effects of sympathetic tone, as illustrated in Figure 2.

Interestingly, the reduction in sympathetic tone caused by SGLT2 inhibitors contributes to the reduction in HF hospitalizations. ${ }^{32}$ In older patients with HF refractory to clinical treatment, ipragliflozin improved sympathetic activity as assessed by 123 I-metaiodobenzylguanidine (MIBG) myocardial scintigraphy, suggesting that this may contribute to the cardioprotective activity of SGLT2 inhibitors. ${ }^{39}$ Additionally, hyperactivation of the sympathetic nervous system is associated with poor prognosis, ventricular arrhythmia or even sudden death. In the EMPA-REG OUTCOME study, empagliflozin was associated with a reduced incidence of sudden death. ${ }^{9}$ Though the precise mechanisms underlying this association have not been fully elucidated, the attenuation of sympathetic hyperactivation caused by SGLT2 inhibitors may reduce the risk of complex arrhythmia and sudden death. The EMBODY ${ }^{40}$ study, which is still ongoing, is investigating whether the effects of SGLT2 on sympathetic tone, through the re-establishment of the balance between sympathetic and parasympathetic tone, could improve survival and reduce the rates of cardiovascular events and sudden death. In this interesting study, patients with diabetes and HF will be randomized to empagliflozin or placebo. The findings from this investigation may provide novel evidence of the effects of this drug class on the reduction of electrical events of the heart. While the exacerbated activation of sympathetic tone is associated with unfavorable vascular remodeling several studies have reported significant reductions in arterial stiffness in patients with DM2 treated with dapagliflozin, ${ }^{41}$ canagliflozin, ${ }^{42}$ or empagliflozin. ${ }^{43}$ This effect is accompanied by improved endothelial function, ${ }^{41}$ promoting reductions in arterial and central pressure with possible effects on the prevention of cardiovascular events. More recently, in a study of 97 patients with DM2, Sposito et al. showed that treatment with dapagliflozin improved endothelial function relative to glibenclamide as determined by Doppler-recorded reactive vasodilation. ${ }^{44}$ These findings indicate that the effect of SGLT1 inhibitors on sympathetic tone and vascular remodeling can contribute to their benefits for patients with HF.

\section{Reduced inflammation}

Inflammation is a major contributor to HF severity. Inflammatory markers are increased in patients with HF and correlate with disease severity ${ }^{45,46}$ in $\mathrm{HF}$ with reduced and preserved EF. ${ }^{47}$ Inflammatory cytokines are known to cause endothelial dysfunction and increase fibrosis and extracellular matrix turnover. SGLT2 inhibitors have the potential to decrease inflammation-associated molecular processes such as extracellular matrix turnover and fibrosis. ${ }^{48}$ This can be illustrated by the fact that dapagliflozin suppresses collagen synthesis in the rat myocardium, demonstrating its antifibrotic effects through the modulation of inflammatory responses. ${ }^{48}$ Despite these findings, it is still not entirely clear how SGLT2 inhibitors modify and modulate the inflammatory process. It is thought that SGLT2 inhibitors initially dampen the macrophage inflammatory response by decreasing the availability of glucose, the preferred energy source for these cells. ${ }^{49}$ The nucleotidebinding oligomerization domain, leucine-rich repeat, and pyrin domain-containing (NLRP3) inflammasome plays an important role in inflammatory mediation. The inflammasome is a complex of interacting proteins that trigger the maturation of pro-inflammatory cytokines to initiate the inflammatory response. This response is amplified by the production of tumor necrosis factor-alpha (TNF-alpha) and the activation of inducible nitric-oxide synthase. Therefore, the inflammasome, or the NLPR3 inflammasome, specifically, may play a central role in the modulation of chronic inflammation, affecting HF progression. ${ }^{50}$ Recent data suggest that SGLT2 inhibitors can reduce NRLP3 inflammasome activation in the heart ${ }^{51,52}$ and that this effect is independent of glucose reduction. It is unclear whether the effects of SGLT2 inhibitors on the NRLP3 inflammasome are direct or indirect. Beta-hydroxybutyrate is an effective blocker of the NLRP3 inflammasome, ${ }^{53}$ and SGLT2 inhibitors increase circulating beta-hydroxybutyrate levels, which may contribute to their mechanism of action.

\section{Prevention of cardiac remodeling}

Cardiac remodeling refers to several structural alterations of the heart muscle, including cardiac hypertrophy, cardiomyocyte inflammation, and fibrosis. These alterations result in increased HR severity. SGLT2 inhibitors have beneficial effects on cardiac remodeling, as documented in several studies. ${ }^{24,30,54-58}$ A randomized trial investigated the effects of a 6-month treatment with empagliflozin or placebo on myocardial modeling in patients with diabetes and coronary artery disease using cardiac magnetic resonance imaging. The study showed that patients treated with empagliflozin exhibited a significant reduction in LV mass relative to those in the placebo group, despite the brevity of the treatment intervention. ${ }^{59}$ This result 


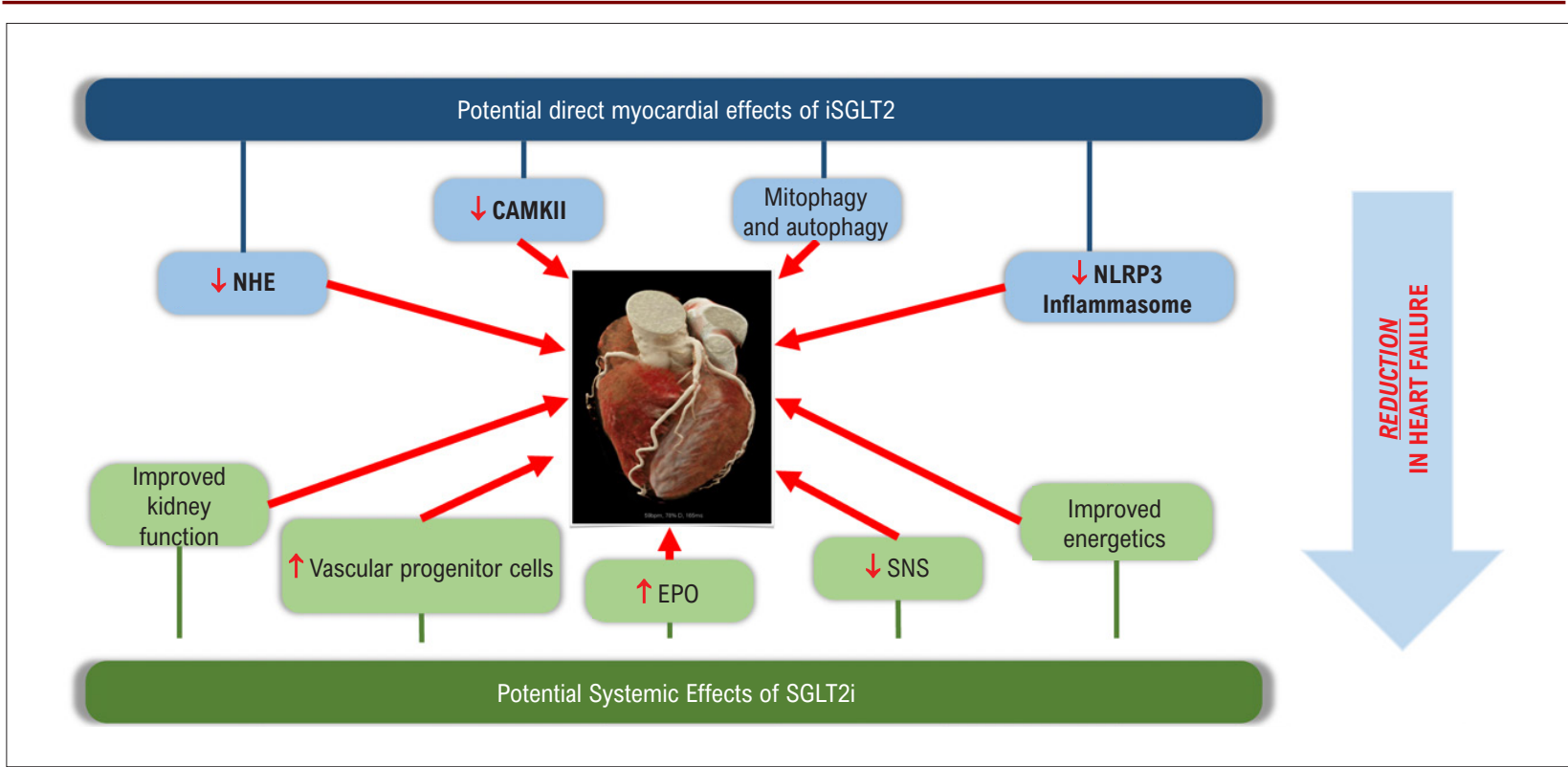

Figure 2- Possible systemic, direct, and indirect effects of iSGLT2. CAMKII: calmodulin-dependent protein kinase; EPO: erythropoietin; NHE: sodium/hydrogen exchange; NLRP3: nucleotide-binding oligomerization domain, leucine-rich repeat, and pyrin domain-containing; iSGLT2: sodium-glucose co-transporter 2 inhibitor; SNS: sympathetic nervous system.

suggests that SGLT2 inhibitors can promote the reverse remodeling of the heart muscle. Although we do not clearly know how SGLT2 inhibitors prevent remodeling, part of this mechanism can be explained by their anti-inflammatory effects, which can reduce fibrosis ${ }^{60,61}$ and, consequently, cardiac remodeling. The inhibition of SGLT2 receptors can therefore reverse the cardiac remodeling observed in HF, reducing the stress on the LV and improving ventricular function.

\section{Suppression of myocardial fibrosis}

As previously mentioned, SGLT2 inhibitors play an important role in the attenuation of myocardial fibrosis, a form of irreversible myocardial damage associated with ventricular dysfunction. HF is accompanied by cardiac remodeling due to the deposition of the extracellular matrix, composed of fibrosis and collagen deposition by cardiac fibroblasts. In patients with DM2, even a 3\% increase in the extracellular volume of the myocardium can lead to a highly significant increase of up to $52 \%$ in the risk of death or hospitalization for HF. It has been proposed that SGLT2 inhibitors may suppress collagen synthesis, activating M2 macrophages and inhibiting myofibroblast differentiation. ${ }^{12,18,19} \mathrm{M} 2$ macrophages secrete interleukin-10 (IL-10), an anti-inflammatory cytokine that plays a crucial role in tissue healing and homeostasis. The reduction in myocardial fibrosis can be demonstrated noninvasively by the assessment of the extracellular volume through advanced imaging methods such as cardiac magnetic resonance, a method that is widely used in contemporary clinical studies. In a study by Mason et al., where patients with diabetes and coronary artery disease were randomized to empagliflozin or placebo for 6 months (Figure 3), the SGLT2 inhibitor led to a $1.4 \%$ reduction in extracellular volume (Figure 4 ) which was associated with a reduction in LV mass. ${ }^{58}$

\section{Reduced ventricular overload}

Another mechanism that may explain the benefits of SGLT2 inhibitors on all-cause mortality in patients with HF and reduced EF could be the reduction in pre- and post-load, and the ensuing improvement in ventricular functioning. ${ }^{12,18,19}$ This occurs because the inhibition of SGLT2 in the proximal tubule promotes natriuresis and glycosuria, resulting in osmotic diuresis, ${ }^{18,60}$ and ultimately reducing ventricular filling pressure and myocardial work. ${ }^{19}$ The natriuretic response, through a tubuloglomerular feedback system, also leads to afferent arteriole vasoconstriction, reducing intraglomerular pressure and possibly explaining the renal preservation associated with the use of SGLT2 inhibitors. ${ }^{18}$ The variation in plasma volume leads to an increase in hematocrit, which was likely responsible for over $50 \%$ of the benefits of these drugs on cardiovascular mortality according to the EMPA-REG OUTCOME ${ }^{19}$ study. Another mechanism that may complement the change in plasma volume and hematocrit is increased erythropoiesis observed in some studies after the start of treatment with SGLT2 inhibitors. Additionally, unlike conventional diuretics, the uricosuric effect that may promote differences in cardiovascular outcomes since serum uric acid is associated with an increased risk of cardiovascular death was present in those using SGLT2 inhibitors. ${ }^{19}$

\section{Repairing vascular function}

Endothelial and smooth vascular muscle dysfunctions contribute to the physiopathology of $\mathrm{HF}^{62}$ and contribute to increased morbidity and mortality rates. SGTL2 inhibitors improve vascular function by decreasing the activation of endothelial cells, inducing direct vascular relaxation. Additionally, SGLT2 inhibition reduces endothelial dysfunction 


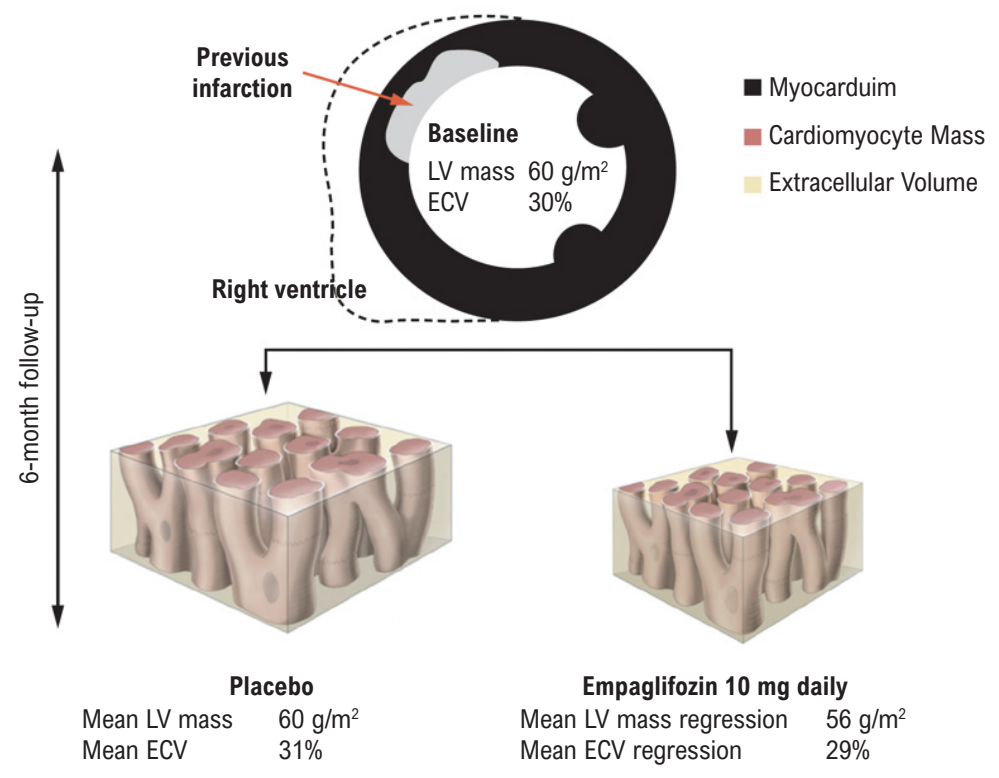

Figure 3 - A 6-month blinded, randomized trial of the effects of empagliflozin vs placebo in patients with type 2 diabetes mellitus (DM2) and coronary artery disease (reproduced with permission from the authors). ${ }^{58} \mathrm{LV}$ : left ventricular; ECV: extracellular volume.

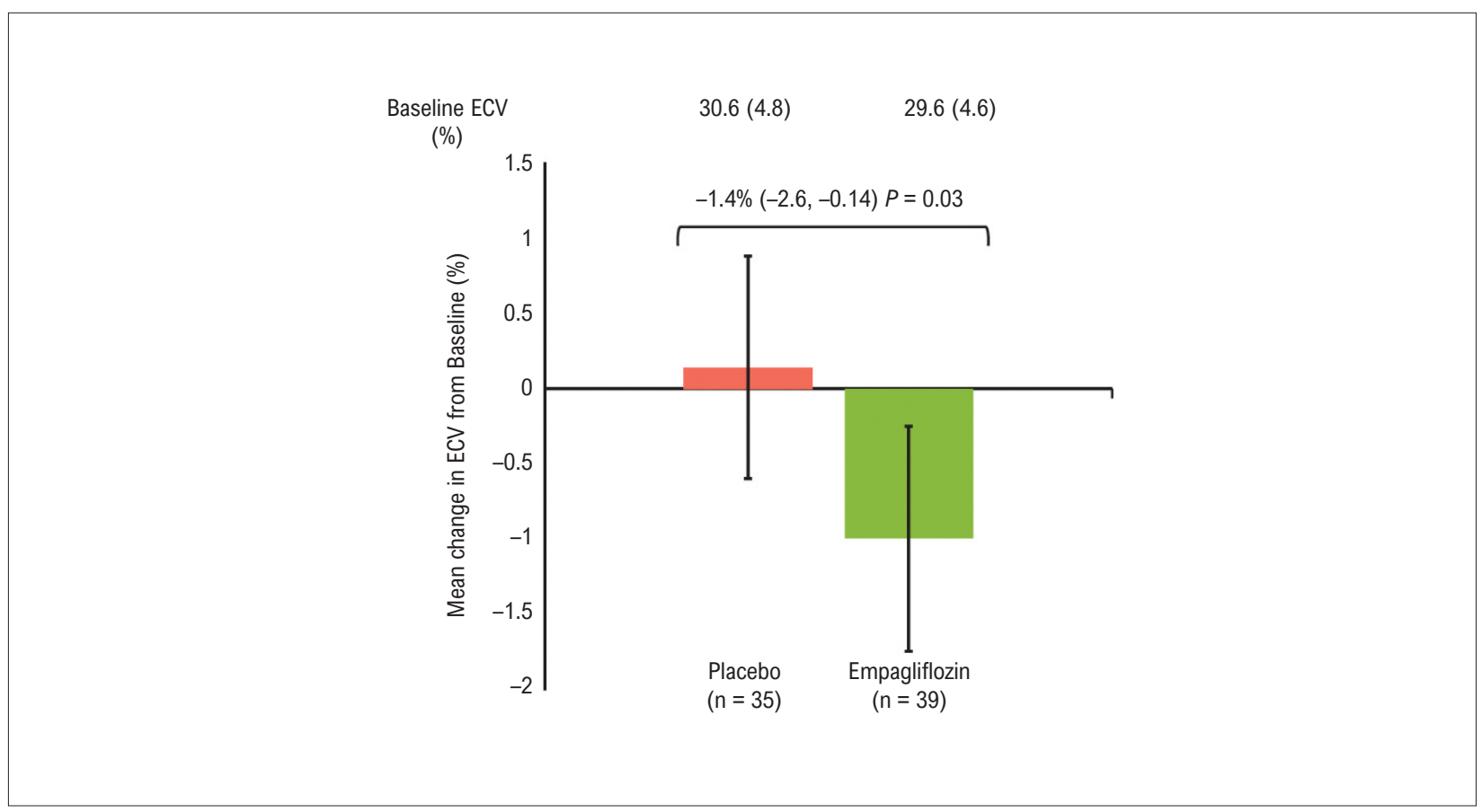

Figure 4 - After 6 months of treatment with empagliflozin, there was a significant reduction in the myocardial extracellular volume of patients with diabetes and coronary artery disease (reproduced with permission from the authors). ${ }^{58} \mathrm{ECV}$ : extracellular volume. 
and molecular alterations associated with early atherogenesis, decreasing arterial stiffness and vascular resistance. ${ }^{63,64}$ Additional benefits of SGLT2 inhibitors include the induction of vasodilation through the activation of protein kinase- $G$ and voltage-gated potassium channels. Together with natriuresis and osmotic diuresis, the direct effects of SGLT2 inhibitors on the vascular system contribute to improved hemodynamic function in patients with HF.

\section{Increasing erythropoietin (EPO) levels}

The increase in hematocrit observed with the use of SGLT2 inhibitors, even in individuals without diabetes, suggests that these medications can increase EPO secretion by the kidneys and ultimately promote erythropoiesis. ${ }^{65}$ This increase in EPO contributes to improvements in cardiomyocyte mitochondrial function, angiogenesis, cell proliferation, and inflammation, in addition to increasing oxygen supply to myocardial tissue. ${ }^{65}$ The EMPA-Heart Cardio Link-6 randomized clinical trial demonstrated that one month of treatment with empagliflozin led to significant increases in EPO levels in patients with diabetes and coronary artery disease. ${ }^{65}$ These patients also exhibited a significant increase in hematocrit and reductions in ferritin and hemoglobin concentration.

\section{Reducing epicardial fat}

Epicardial adipose tissue is the visceral fat that surrounds the heart and comes into direct contact with the coronary tunica. The attenuation of epicardial adipose tissue as demonstrated on computed tomography can indicate the presence of inflammatory alterations and coronary neovascularization and is therefore associated with an increased risk of cardiovascular events. ${ }^{20}$ SGLT2 inhibitors reduce the accumulation of adipose tissue and perivascular inflammation, minimizing the secretion of leptin and its effects on cardiac fibrosis. ${ }^{66}$ In patients with both diabetes and coronary artery disease, SGLT2 inhibition reduces the mass of epicardial adipose tissue and bioactive molecules such as TNF-alpha and the type 1 plasminogen activator inhibitor. This can therefore attenuate inflammation and even the remodeling associated with $\mathrm{HF}^{67}$

\section{Increased vascular progenitor cells}

Another mechanism that could explain the cardioprotective effect of SGLT2 inhibitors is the restoration of vascular repair through the mobilization of vascular progenitor cells in the bone marrow. Tissue revascularization requires a network of circulating cells to work together to mediate microvascular growth. There is evidence to suggest that SGLT2 inhibition may affect the balance of circulating progenitor vascular cells, monocytes, and inflammatory cells in patients with diabetes and established cardiovascular disease. ${ }^{18}$ In a previous study, Hess et al. ${ }^{68}$ observed that treatment with empagliflozin was associated with a reduction in M1 proinflammatory macrophages, and a subsequent increase in the number of polarized M2 macrophages which are associated with an anti-inflammatory response (Th2). Previous studies have demonstrated that phlorizin-induced SGLT2 inhibition increases antioxidant production, and the administration of empagliflozin reduces the production of NOX1, an important component of oxidative stress in the peripheral vasculature of rats with diabetes. ${ }^{69}$ Systemic reductions in oxidative stress promote the maturation of progenitor cells in the bone marrow. ${ }^{70}$ It has been proposed that the administration of SGLT2 inhibitors leads to an increase in the release of angiogenic progenitor cells from the bone marrow. ${ }^{68}$

\section{Improved metabolism and cardiac bioenergetics}

Fatty acids are an important energy source for healthy heart tissue; however, HF is associated with impaired cardiac fatty acid oxidation. In these cases, the heart utilizes ketone bodies as an alternative energy source through enzymes such as beta-hydroxybutyrate dehydrogenase (BHD1). SGLT2 inhibitors can increase the production of ketone bodies, especially beta-hydroxybutyrate, increasing the energy supply to the heart muscle..$^{18}$ In this way, the change in metabolic substrate contributes to increased efficiency and myocardial contractility. The increased ketone production is also believed to reduce acetyl CoA levels, which, in turn, reduce the hyperacetylation of mitochondrial enzymes, improving mitochondrial energy production. ${ }^{12}$

\section{Inhibition of sodium/hydrogen exchangers}

Another explanation for the effects of SGLT2 inhibitors on HF involves their interaction with type 1 (NHE1) and 3 (NHE3) sodium/hydrogen exchangers. NHE1 is found in myocardial and vascular tissues, and when activated, increases intracellular sodium and calcium levels, the first of which is associated with HF and cardiomyocyte injury. NHE3 is found in the kidneys and promotes sodium reabsorption and subsequent water retention. ${ }^{12}$ SGLT2 inhibitors are believed to directly inhibit NHE1 and promote natriuresis by reducing NHE3 expression. This pathway may contribute to the explanation of the close link between diabetes and HF. They are stimulated by elevated glucose and insulin levels in diabetes and by the exacerbated neurohumoral activation in HF. Therefore, patients with decompensated HF can also experience an exacerbation of diabetes. ${ }^{12,46}$ In conclusion, the inhibition of NHE1 and NHE3 may be an important part of the cardiac and renal mechanisms through which SGLT2 inhibitors treat HF. ${ }^{18}$

\section{Reduced leptin levels}

Leptin, a protein associated with energy homeostasis, is a major contributor to the development of HF in individuals with diabetes. This protein stimulates SGLT2 receptors in the kidneys, causing sodium retention and influencing inflammation and cardiac fibrosis. SGLT2 inhibitors can suppress the effects of leptin in several ways. The reduction in visceral adipose tissue lowers leptin levels, while the inhibition of SGLT2 receptors in the kidneys reduces sodium absorption. ${ }^{18}$ SGLT2 inhibitors also increase overall adiponectin levels, decreasing inflammatory action through TNF-alpha inhibition. Adiponectin is associated with reductions in body fat, which it promotes by inhibiting SGLT2. 


\section{Prevention of ischemia/reperfusion injury}

Cell injury, necrosis, and programmed cell death (apoptosis, necroptosis, autophagy) are important physiopathological characteristics of several maladaptive processes in the heart, including myocardial ischemia and HF. ${ }^{71}$ Recent experimental evidence suggests that SGLT2 inhibition has cardioprotective effects against ischemia/reperfusion injuries. The authors of these studies hypothesize that the improved cardiovascular survival observed after treatment with SGLT2 inhibitors is associated with their direct cytoprotective effects which include protection against myocardial ischemia/reperfusion injury. The first demonstration of the cardioprotective effects of canagliflozin against ischemia/reperfusion injury in diabetic and non-diabetic animals was obtained in a study of Langendorff-perfused hearts from diabetic and non-diabetic rats treated with canagliflozin for a 4-week period, who showed smaller infarcts than those in comparison conditions. ${ }^{61}$ These results also suggest that SGLT2 inhibitors may be introduced as a cardioprotective intervention in highrisk cardiovascular patients, irrespective of diabetes status. ${ }^{61}$ The beneficial effect of SGLT2 on ischemia/reperfusion injury is associated with a reduction in the activity of calmodulin kinase II, resulting in increased calcium flux in the sarcoplasmic reticulum and increased contractility. However, it is not yet clear if this effect occurs in humans.

\section{Reducing hyperuricemia}

Plasma uric acid has a negative effect on the prognosis of HF. ${ }^{72}$ Uric acid serum levels are intimately related to insulin resistance markers. Patients with DM2 are known to have lower serum uric acid levels than the general population, and to date, this has been attributed to the osmotic diuresis caused by elevated serum glucose. Slight reductions in plasma uric acid have been observed after treatment with SGLT2 inhibitors. ${ }^{73}$ This can be attributed to the increase in glycosuria in the proximal tubules since SGLT2 inhibitors promote uric acid secretion. ${ }^{74}$ However, the issue of whether reduced hyperuricemia due to SGLT2 inhibition is a marker or cause of its effects remains open for discussion.

\section{References}

1. Khan MS, Vaduganathan M. What makes sodium-glucose co-transporter-2 inhibitors stand out in heart failure? Curr Diab Rep. 2020;20(11):63. doi: 10.1007/s11892-020-01347-3.

2. Comitê Coordenador da Diretriz de Insuficiência Cardíaca, Rohde LEP, Montera MW, Bocchi EA, Clausell NO, Albuquerque DC, et al. Diretriz Brasileira de Insuficiência Cardíaca Crônica e Aguda. Arq Bras Cardiol. 2018;111(3):436-539. doi: 10.5935/abc.20180190.

3. Ponikowski P, Voors AA, Anker SD, Bueno H, Cleland JGF, Coats AJS, et al. 2016 ESC Guidelines for the diagnosis and treatment of acute and chronic heart failure: The Task Force for the diagnosis and treatment of acute and chronic heart failure of the European Society of Cardiology (ESC) Developed with the special contribution of the Heart Failure Association (HFA) of the ESC. Eur HeartJ. 2016;37(27):2129-2200. doi: 10.1093/eurheartj/ehw128.

\section{Conclusions}

Several studies have demonstrated the benefits of SGLT2 inhibitors on cardiovascular outcomes. Yet the precise mechanisms behind the cardioprotective effects of SGLT2 inhibitors have not been entirely established. Several such mechanisms have been proposed, including reduced sympathetic activity, improved cardiovascular hemodynamics, and bioenergetic availability. These are all complex, interdependent factors that potentiate one another. As such, further studies are needed to shed light on the effects of SGLT2 inhibitors in the context of HF. SGLT2 inhibitors are promising drugs with proven effectiveness in the treatment of several chronic illnesses, starting with DM2 and extending to HF with reduced EF in 2019, and, as recently established by the DAPA-CKD study, consistent benefits on kidney function.

\section{Author Contributions}

Conception and design of the research, Acquisition of data, Writing of the manuscript and Critical revision of the manuscript for intellectual content: Martins CNG, Bau AA, Silva LM, Coelho-Filho OR

\section{Potential Conflict of Interest}

No potential conflict of interest relevant to this article was reported.

\section{Sources of Funding}

There were no external funding sources for this study.

\section{Study Association}

This study is not associated with any thesis or dissertation work.

\section{Ethics approval and consent to participate}

This article does not contain any studies with human participants or animals performed by any of the authors.

4. Martens P, Mathieu C, Verbrugge FH. Promise of SGLT2 inhibitors in heart failure: diabetes and beyond. Curr Treat Options Cardiovasc Med. 2017;19(3):23. doi: 10.1007/s11936-017-0522-x.

5. Giorda CB. 2019 ESC Guidelines on diabetes, pre-diabetes and cardiovascular diseases: what's new? The diabetologist's point of view. G Ital Cardiol. 2020;21(4):252-55. doi: 10.1714/3328.32983.

6. Khan MS, Fonarow GC, Greene SJ. Sodium-glucose co-transporter 2 inhibitors for heart failure: clinical trial efficacy and clinical practice effectiveness. Eur J Heart Fail. 2021. doi: 10.1002/ejhf.2170.

7. Wiviott SD, Raz I, Bonaca MP, Mosenzon O, Kato ET, Cahn A, et al. Dapagliflozin and cardiovascular outcomes in type 2 diabetes. N Engl J Med. 2019;380(4):347-57. doi: 10.1056/NEJMoa1812389. 
8. Neal B, Perkovic V, Matthews DR. Canagliflozin and Cardiovascular and Renal Events in Type 2 Diabetes. N Engl J Med. 2017;377(21):2099. doi: 10.1056/NEJMc1712572.

9. Zinman B, Wanner C, Lachin JM, Fitchett D, Bluhmki E, Hantel S, et al. Empagliflozin, cardiovascular outcomes, and mortality in type 2 diabetes. N Engl J Med. 2015 Nov 26;373(22):2117-28. doi: 10.1056/ NEJMoa1504720.

10. McMurray JJV, Solomon SD, Inzucchi SE, Kober L, Kosiborod MN, Martinez FA, et al. Dapagliflozin in patients with heart failure and reduced ejection fraction. N Engl J Med. 2019;381(21):1995-2008. doi: 10.1056/ NEJMoa1911303.

11. Packer M, Anker SD, Butler J, Filippatos G, Pocock SJ, Carson P, et al. Cardiovascular and renal outcomes with empagliflozin in heart failure. N Engl J Med. 2020;383(15):1413-24. doi: 10.1056/NEJMoa2022190.

12. Nightingale B. A Review of the proposed mechanistic actions of sodium glucose cotransporter- 2 inhibitors in the treatment of heart failure. Cardiol Res. 2021;12(2):60-6. doi: 10.14740/cr1221.

13. Nissen SE, Wolski K. Effect of rosiglitazone on the risk of myocardial infarction and death from cardiovascular causes. N Engl J Med. 2007;356(24):2457-71. doi: 10.1056/NEJMoa072761.

14. Lincoff AM, Wolski K, Nicholls SJ, Nissen SE. Pioglitazone and risk of cardiovascular events in patients with type 2 diabetes mellitus: a metaanalysis of randomized trials. JAMA. 2007;298(10):1180-8. doi: 10.1001/ jama.298.10.1180.

15. Lipscombe LL, Gomes T, Lévesque LE, Hux JE, Juurlink DN, Alter DA. Thiazolidinediones and cardiovascular outcomes in older patients with diabetes. JAMA. 2007;298(22):2634-43. doi: 10.1001/jama.298.22.2634.

16. Scirica BM, Braunwald E, Raz I, Cavender MA, Morrow DA, Jarolim P, et al. Heart failure, saxagliptin, and diabetes mellitus: observations from the SAVOR-TIMI 53 randomized trial. Circulation. 2014;130(18):1579-88. doi: 10.1161/CIRCULATIONAHA.114.010389.

17. Udell JA, Bhatt DL, Braunwald E, Cavender MA, Mosenzon O, Steg PG, etal. Saxagliptin and cardiovascular outcomes in patients with type 2 diabetes and moderate or severe renal impairment: observations from the SAVOR-TIMI 53 Trial. Diabetes Care. 2015;38(4):696-705. doi: 10.2337/dc14-1850.

18. Verma S, MCMurray JJV. SGLT2 inhibitors and mechanisms of cardiovascular benefit: a state-of-the-art review. Diabetologia. 2018;61(10):2108-17. doi: 10.1007/s00125-018-4670-7.

19. Inzucchi SE, Zinman B, Fitchett D, Wanner C, Ferrannini E, Schumacher $M$, et al. how does empagliflozin reduce cardiovascular mortality? insights from a mediation analysis of the EMPA-REG outcome trial. Diabetes Care. 2018:41(2):356-63. doi: 10.2337/dc17-1096.

20. Raggi P, Gadiyaram V, Zhang C, Chen Z, Lopaschuk G, Stillman AE. Statins reduce epicardial adipose tissue attenuation independent of lipid lowering: a potential pleiotropic effect. J Am Heart Assoc. 2019;8(12):e013104. doi: 10.1161/JAHA.119.013104.

21. Cai X, Yang W, Gao X, Chen Y, Zhou L, Zhang S, et al. The association between the dosage of SGLT2 inhibitor and weight reduction in type 2 diabetes patients: a meta-analysis. Obesity (Silver Spring). 2018;26(1):7080. doi: 10.1002/oby.22066.

22. Lee PC, Ganguly S, Goh SY. Weight loss associated with sodiumglucose cotransporter-2 inhibition: a review of evidence and underlying mechanisms. Obes Rev. 2018;19(12):1630-41. doi: 10.1111/obr.12755.

23. Sattar N. Revisiting the links between glycaemia, diabetes and cardiovascular disease. Diabetologia. 2013;56(4):686-95. doi: 10.1007/s00125-012-2817-5.

24. Connelly KA, Zhang Y, Visram A, Advani A, Batchu SN, Desjardins JF, et al. Empagliflozin improves diastolic function in a nondiabetic rodent model of heart failure with preserved ejection fraction. JACC Basic Transl Sci. 2019;4(1):27-37. doi: 10.1016/j.jacbts.2018.11.010.
25. Cannon CP, Perkovic V, Agarwal R, Baldassarre J, Bakris G, Charytan DM et al. Evaluating the effects of canagliflozin on cardiovascular and renal events in patients with type 2 diabetes mellitus and chronic kidney disease according to baseline $\mathrm{HbA} 1 \mathrm{c}$, including those with $\mathrm{HbA} 1 \mathrm{c}<7 \%$ : results from the CREDENCE trial. Circulation. 2020;141(5):407-10. doi: 10.1161/ CIRCULATIONAHA.119.044359.

26. Mazidi M, Rezaie P, Gao HK, Kengne AP. Effect of sodium-glucose cotransport- 2 inhibitors on blood pressure in people with type 2 diabetes mellitus: a systematic review and meta-analysis of 43 randomized control trials with 22528 patients. J Am Heart Assoc. 2017;6(6):e004007. doi: 10.1161/JAHA.116.004007.

27. Ferrannini E, Baldi S, Frascerra S, Astiarraga B, Barsotti E, Clerico A, et al Renal handling of ketones in response to sodium-glucose cotransporter 2 inhibition in patients with type 2 diabetes. Diabetes Care. 2017;40(6):7716. doi: $10.2337 / \mathrm{dc} 16-2724$.

28. Weber MA, Mansfield TA, Cain VA, Iqbal N, Parikh S, Ptaszynska A. Blood pressure and glycaemic effects of dapagliflozin versus placebo in patients with type 2 diabetes on combination antihypertensive therapy: a randomised, double-blind, placebo-controlled, phase 3 study. Lancet Diabetes Endocrinol. 2016;4(3):211-20. doi: 10.1016/S22138587(15)00417-9.

29. Kario K, Böhm M, Mahfoud F, Townsend RR, Weber MA, Patel M, et al Twenty-four-hour ambulatory blood pressure reduction patterns after renal denervation in the SPYRAL HTN-OFF MED trial. Circulation. 2018;138(15):1602-4. doi: 10.1161/CIRCULATIONAHA.118.035588.

30. Heerspink HJL, de Zeeuw D, Wie L, Leslie B, List J. Dapagliflozin a glucoseregulating drug with diuretic properties in subjects with type 2 diabetes. Diabetes Obes Metab. 2013;15(9):853-62. doi: 10.1111/dom.12127.

31. Hallow KM, Helmlinger G, Greasley PJ, McMurray JJV, Boulton DW. Why do SGLT2 inhibitors reduce heart failure hospitalization? A differential volume regulation hypothesis. Diabetes Obes Metab. 2018;20(3):479-87. doi: 10.1111/dom.13126.

32. Sano M. A new class of drugs for heart failure: SGLT2 inhibitors reduce sympathetic overactivity. J Cardiol. 2018;71(5):471-6. doi: 10.1016/j. jjcc.2017.12.004

33. Vecchione C, Argenziano L, Fratta L, Pompeo F, Trimarco B. Sympathetic nervous system and hypertension in diabetic patients. Diabetes Nutr Metab. 2000;13(6):327-31.

34. Lim K, Jackson KL, Sata Y, Head GA. Factors responsible for obesity-related hypertension. Curr Hypertens Rep. 2017;19(7):53. doi: 10.1007/s11906017-0750-1.

35. Toschi-Dias E, Rondon MUPB, Cogliati C, Paolocci N, Tobaldini E, Montano $\mathrm{N}$. contribution of autonomic reflexes to the hyperadrenergic state in heart failure. Front Neurosci. 2017;11:162. doi: 10.3389/fnins.2017.00162.

36. van Bilsen M, Patel HC, Bauersachs J, Böhm M, Borggrefe M, Brutsaert D, et al. The autonomic nervous system as a therapeutic target in heart failure: a scientific position statement from the Translational Research Committee of the Heart Failure Association of the European Society of Cardiology. Eur J Heart Fail. 2017;19(11):1361-78. doi: 10.1002/ejhf.921.

37. Wan N, Rahman A, Hitomi H, Nishiyama A. The effects of sodium-glucose cotransporter 2 inhibitors on sympathetic nervous activity. Front Endocrinol (Lausanne). 2018;9:421. doi: 10.3389/fendo.2018.00421.

38. Jordan J, Tank J, Heusser K, Heise T, Wanner C, Heer M, et al. The effect of empagliflozin on muscle sympathetic nerve activity in patients with type II diabetes mellitus. J Am Soc Hypertens. 2017;11(9):604-12. doi: 10.1016/j. jash.2017.07.005

39. Kiuchi S, Hisatake S, Kabuki T, Fujii T, Oka T, Dobashi S, et al. Long-term use of ipragliflozin improved cardiac sympathetic nerve activity in a patient with heart failure: a case report. Drug Discov Ther. 2018;12(1):51-4. doi: 10.5582/ddt.2017.01069. 
40. Kubota Y, Yamamoto T, Tara S, Tokita Y, Yodogawa K, Iwasaki Y, et al. Effect of Empagliflozin versus placebo on cardiac sympathetic activity in acute myocardial infarction patients with type 2 diabetes mellitus: rationale. Diabetes Ther. 2018;9(5):2107-16. doi: 10.1007/s13300-018-0480-7.

41. Solini A, Giannini L, Seghieri M, Vitolo E, Taddei S, Ghiadoni L, et al. Dapagliflozin acutely improves endothelial dysfunction, reduces aortic stiffness and renal resistive index in type 2 diabetic patients: a pilot study. Cardiovasc Diabetol. 2017;16(1):138. doi: 10.1186/s12933-017-0621-8.

42. Ramirez AJ, Sanchez MJ, Sanchez RA. Diabetic patients with essential hypertension treated with amlodipine: blood pressure and arterial stiffness effects of canagliflozin or perindopril. J Hypertens. 2019;37(3):636-42. doi: 10.1097/HJH.0000000000001907.

43. Chilton R, Tikkanen I, Cannon CP, Crowe S, Woerle HJ, Broedl UC, et al. Effects of empagliflozin on blood pressure and markers of arterial stiffness and vascular resistance in patients with type 2 diabetes. Diabetes Obes Metab. 2015;17(12):1180-93. doi: 10.1111/dom.12572.

44. Sposito AC, Breder I, Soares AAS, Kimura-Medorima ST, Munhoz DB, Cintra RMR, et al. Dapagliflozin effect on endothelial dysfunction in diabetic patients with atherosclerotic disease: a randomized active-controlled trial. Cardiovasc Diabetol. 2021;20(1):74. doi: 10.1186/s12933-021-01264-z.

45. Dick SA, Epelman S. Chronic heart failure and inflammation: what do we really know? Circ Res. 2016;119(1):159-76. doi: 10.1161/CIRCRESAHA.116.308030.

46. MehtaJL, Pothineni NV. Inflammation in heartfailure: the holygrail? Hypertension. 2016;68(1):27-9. doi: 10.1161/HYPERTENSIONAHA.116.07307.

47. Briasoulis A, Androulakis E, Christophides T, Tousoulis D. The role of inflammation and cell death in the pathogenesis, progression and treatment of heart failure. Heart Fail Rev. 2016;21(2):169-76. doi: 10.1007/s10741-016-9533-z.

48. Lee TM, Chang NC, Lin SZ. Dapagliflozin, a selective SGLT2 Inhibitor, attenuated cardiac fibrosis by regulating the macrophage polarization via STAT3 signaling in infarcted rat hearts. Free Radic Biol Med. 2017;104:298310. doi: 10.1016/j.freeradbiomed.2017.01.035

49. Rotkvić PG, Berković MC, Bulj N, Rotkvić L. Minireview: are SGLT2 inhibitors heart savers in diabetes? Heart Fail Rev. 2020;25(6):899-905. doi: 10.1007/s10741-019-09849-3.

50. Butts B, Gary RA, Dunbar SB, Butler J. The importance of nlrp3 inflammasome in heart failure. J Card Fail. 2015;21(7):586-93. doi: 10.1016/j.cardfail.2015.04.014

51. Ye Y, Jia X, Bajaj M, Birnbaum Y. Dapagliflozin attenuates $\mathrm{Na}+/ \mathrm{H}+$ Exchanger-1 in cardiofibroblasts via AMPK activation. Cardiovasc Drugs Ther. 2018;32(6):553-8. doi: 10.1007/s10557-018-6837-3.

52. Byrne NJ, Matsumura N, Maayah ZH, Ferdaoussi M, Takahara S, Darwesh AM, et al. Empagliflozin blunts worsening cardiac dysfunction associated with reduced NLRP3 (Nucleotide-Binding Domain-Like Receptor Protein 3) inflammasome activation in heart failure. Circ Heart Fail. 2020;13(1):e006277. doi: 10.1161/ CIRCHEARTFAILURE.119.006277.

53. Youm YH, Nguyen KY, Grant RW, Goldberg EL, Bodogai M, Kim D, et al. The ketone metabolite $\beta$-hydroxybutyrate blocks NLRP3 inflammasomemediated inflammatory disease. Nat Med. 2015;21(3):263-9. doi: $10.1038 / \mathrm{nm} .3804$.

54. Byrne NJ, Parajuli N, Levasseur JL, Boisvenue J, Beker DL, Masson G, et al. Empagliflozin prevents worsening of cardiac function in an experimental model of pressure overload-induced heart failure. JACC Basic Transl Sci. 2017;2(4):347-54. doi: 10.1016/j.jacbts.2017.07.003.

55. Shi L, Zhu D, Wang S, Jiang A, Li F. Dapagliflozin attenuates cardiac remodeling in mice model of cardiac pressure overload. Am J Hypertens. 2019;32(5):452-9. doi: 10.1093/ajh/hpz016.

56. Verma S, Garg A, Yan AT, Gupta AK, Al-Omran M, Sabongui A, et al. Effect of Empagliflozin on left ventricular mass and diastolic function in individuals with diabetes: an important clue to the EMPA-REG OUTCOME trial? Diabetes Care. 2016;39(12):e212-e213. doi: 10.2337/dc16-1312.
57. Esterline RL, Vaag A, Oscarsson J, Vora J. Mechanisms in endocrinology: SGLT2 inhibitors: clinical benefits by restoration of normal diurnal metabolism? Eur J Endocrinol. 2018;178(4):R113-R125. doi: 10.1530/ EJE-17-0832.

58. Mason T, Coelho-Filho OR, Verma S, Chowdhury B, Zuo F, Quan A, et al. Empagliflozin reduces myocardial extracellular volume in patients with type 2 diabetes and coronary artery disease. JACC Cardiovasc Imaging. 2021:S1936-878X(20)30939-6. doi: 10.1016/j.jcmg.2020.10.017.

59. Verma S, Mazer CD, Yan AT, Mason T, Garg V, Teoh H, et al. Effect of Empagliflozin on left ventricular mass in patients with type 2 diabetes mellitus and coronary artery disease: the EMPA-HEART CardioLink-6 randomized clinical trial. Circulation. 2019;140(21):1693-702. doi: 10.1161/ CIRCULATIONAHA.119.042375.

60. Kang S, Verma S, Hassanabad AF, Teng G, Belke DD, Dundas JA, et al. Direct effects of Empagliflozin on extracellular matrix remodelling in human cardiac myofibroblasts: novel translational clues to explain EMPA-REG OUTCOME results. Can J Cardiol. 2020;36(4):543-53. doi: 10.1016/j.cjca.2019.08.033.

61. Lim VG, Bell RM, Arjun S, Kolatsi-Joannou M, Long DA, Yellon DM. SGLT2 inhibitor, canagliflozin, attenuates myocardial infarction in the diabetic and nondiabetic heart. JACC Basic Transl Sci. 2019;4(1):15-26. doi: 10.1016/j. jacbts.2018.10.002

62. Endemann DH, Schiffrin EL. Endothelial dysfunction. J Am Soc Nephrol. 2004;15(8):1983-92. doi: 10.1097/01.ASN.0000132474.50966.DA.

63. Juni RP, Kuster DWD, Goebel M, Helmes M, Musters RJP, van der Velden J, et al. Cardiac microvascular endothelial enhancement of cardiomyocyte function is impaired by inflammation and restored by Empagliflozin. JACC Basic Transl Sci. 2019;4(5):575-91. doi: 10.1016/j.jacbts.2019.04.003.

64. Patel AR, Kuvin JT, Pandian NG, Smith JJ, Udelson JE, Mendelsohn ME, et al. Heart failure etiology affects peripheral vascular endothelial function after cardiac transplantation. J Am Coll Cardiol. 2001;37(1):195-200. doi: 10.1016/s0735-1097(00)01057-3.

65. Mazer CD, HareGMT, Connelly PW, GilbertRE, Shehata N, Quan A, etal. Effect of Empagliflozin on erythropoietin levels, iron stores, and red blood cell morphology in patients with type 2 diabetes mellitus and coronary artery disease. Circulation. 2020;141(8):704-7. doi: 10.1161/CIRCULATIONAHA.119.044235.

66. Sano M, Takei M, Shiraishi Y, Suzuki Y. Increased hematocrit during sodium-glucose cotransporter 2 inhibitor therapy indicates recovery of tubulointerstitial function in diabetic kidneys. J Clin Med Res. 2016;8(12):844-7. doi: 10.14740/jocmr2760w.

67. Sato T, Aizawa Y, Yuasa S, Kishi S, Fuse K, Fujita S, et al. The effect of dapagliflozin treatment on epicardial adipose tissue volume. Cardiovasc Diabetol. 2018;17(1):6. doi: 10.1186/s12933-017-0658-8.

68. Hess DA, Terenzi DC, Trac JZ, Quan A, Mason T, Al-Omran M, et al. SGLT2 Inhibition with Empagliflozin increases circulating provascular progenitor cells in people with type 2 diabetes mellitus. Cell Metab. 2019;30(4):609-13. doi: 10.1016/j.cmet.2019.08.015.

69. Oelze M, Kröller-Schön S, Welschof P, Jansen T, Hausding M, Mikhed Y, et al. The sodium-glucose co-transporter 2 inhibitor empagliflozin improves diabetes-induced vascular dysfunction in the streptozotocin diabetes rat model by interfering with oxidative stress and glucotoxicity. PLoS One. 2014;9(11):e112394. doi: 10.1371/journal.pone.0112394.

70. Terenzi DC, Trac JZ, Teoh H, Gerstein HC, Bhatt DL, Al-Omran M, et al. Vascular regenerative cell exhaustion in diabetes: translational opportunities to mitigate cardiometabolic risk. Trends Mol Med. 2019;25(7):640-55. doi: 10.1016/j.molmed.2019.03.006.

71. Moe GW, Marín-García J. Role of cell death in the progression of heart failure. Heart Fail Rev. 2016;21(2):157-67. doi: 10.1007/s10741-016-9532-0.

72. Hare JM, Johnson RJ. Uric acid predicts clinical outcomes in heart failure: insights regarding the role of xanthine oxidase and uric acid in disease pathophysiology. Circulation. 2003;107(15):1951-3. doi: 10.1161/01. CIR.0000066420.36123.35. 
73. Schernthaner G, Gross JL, Rosenstock J, Guarisco M, Fu M, Yee J, et al. Canagliflozin compared with sitagliptin for patients with type 2 diabetes who do nothave adequate glycemic control with metformin plussulfonylurea: a 52-week randomized trial. Diabetes Care. 2013;36(9):2508-15. doi: 10.2337/dc12-2491.
74. Chino Y, Samukawa Y, Sakai S, Nakai Y, Yamaguchi J, Nakanishi T, etal. SGLT2 inhibitor lowers serum uric acid through alteration of uric acid transport activity in renal tubule by increased glycosuria. Biopharm Drug Dispos. 2014;35(7):391-404. doi: 10.1002/bdd.1909. 